\title{
Under the Tucson sun: A meeting in the desert on mRNA decay
}

\author{
KRISTIAN E. BAKER ${ }^{1}$ and CIARÁN CONDON ${ }^{2}$ \\ ${ }^{1}$ Howard Hughes Medical Institute, Department of Molecular \& Cellular Biology, Tucson, Arizona 85745, USA \\ ${ }^{2}$ Institut de Biologie Physico-Chimique, 75005 Paris, France
}

\section{INTRODUCTION}

Scientists from all over the world gathered this past June in Tucson, Arizona, for a FASEB-sponsored meeting organized by George Mackie (Univ. of British Columbia) and Roy Parker (Univ. of Arizona) highlighting new developments in the area of posttranscriptional gene regulation by mRNA degradation. The researchers in attendance represented two biological realms, eubacteria and eukaryotes, and while the molecular players, pathways, and regulation of mRNA turnover in these two phyla are clearly distinct, recurrent themes were unmistakable. Regardless of the organism, data were presented reinforcing our understanding that mRNA degradation in all systems is modulated by trans-acting factors, ribonucleolytic or not, in response to cis-acting RNA sequences. Furthermore, the adjustment of trans-effector activity and the accessibility of the cis-acting regulatory sequences is used by both prokaryotes and eukaryotes to provide additional layers of complexity to the process. A role for RNA polyadenylation in destabilizing eukaryotic RNA, reminiscent of the decay of stable RNAs in bacteria, was recently uncovered, and in both organisms, alternative fates for mRNAs engaged in translation were presented. Structural information for ribonucleases and accessory RNA decay proteins is proving to offer insight into catalytic function and possible regulatory mechanisms governing mRNA turnover. Moreover, the characterization of mRNA degradation in new model organisms, both bacterial and eukaryotic, is leading to both the confirmation of existing decay pathways and the discovery of new ones.

\section{mRNA STABILIZATION BY CIS-ACTING ELEMENTS: PARALLELS BETWEEN BACTERIA AND EUKARYOTES}

The influence of the extreme $5^{\prime}$ terminus on protecting mRNA against degradation is well documented for both

Reprint requests to: Kristian E. Baker, Howard Hughes Medical Institute, Department of Molecular \& Cellular Biology, 1007 East Lowell Street, Room 403 Life Sciences South, Tucson, AZ 85745, USA; e-mail: kebaker@email.arizona.edu; fax: (520)621-4524.

Article and publication are at http://www.rnajournal.org/cgi/doi/ 10.1261/rna.7163104. bacteria and eukaryotes. In Escherichia coli, the 5'-terminal triphosphate acts to stabilize the mRNA from a decay-initiating RNA cleavage event catalyzed by the endoribonuclease, RNase E (Mackie 1998, 2000). RNase E, in fact, displays a strong preference for monophosphorylated RNA substrates, ensuring that once decay of an mRNA is initiated, continued degradation of the $3^{\prime}$ cleavage product is preferred over an intact pppRNA. The sensitivity of RNase E to the phosphorylation status of the RNA $5^{\prime}$ terminus was presumed to be correlated simply to the relative affinity of the enzyme for monophosphorylated versus triphosphorylated mRNA. Work presented by Joel Belasco (NYU) suggests, rather, that $5^{\prime}$ monophosphates serve a more participatory role in mRNA stability by increasing the catalytic efficiency and cleavage site specificity of RNase E and its homolog, RNase G (Jiang and Belasco 2004). Interestingly, both RNases must assemble into their multimeric state for this effect on catalysis, suggesting a model whereby $5^{\prime}$-end binding by one RNase $\mathrm{E}$ subunit, for example, can induce conformational changes that, depending on the status of the 5 ' end of the mRNA, differentially enhance RNA cleavage by a second subunit. The analysis of kinetic parameters for RNase E was greatly facilitated by the development of a continuous, real-time assay using synthetic fluorogenic RNA substrates (Jiang and Belasco 2004). The use of fluorescence resonance energy transfer (FRET) methodology allows for initial rate measurements that occur on a timescale that could not be accessed with conventional biochemical methods.

In eukaryotes, the 7-methyl guanosine cap structure present on the mRNA $5^{\prime}$ terminus is inhibitory to $5^{\prime}-3^{\prime}$ exonucleolytic decay catalyzed in yeast by Xrn1p, and, moreover, its removal from the mRNA by the decapping complex, Dcp1/2, often represents the rate-limiting step in mRNA turnover (see Coller and Parker 2004). While the activity of Dcp1/2 is coupled to prior deadenylation of the mRNA, the scavenger decapping enzyme, DcpS, preferentially hydrolyzes cap structures from terminal oligoribonucleotides generated by the exosome-mediated $3^{\prime}-5^{\prime}$ pathway of decay (Liu et al. 2002). Findings presented by Mike Kiledjian (Rutgers) provide an explanation for the observa- 
tion that intact mRNAs are not substrates for DcpS. Kiledjian showed that the affinity and subsequent activity of mammalian DcpS for $5^{\prime}$ cap structures is sensitive to the length of the RNA in which the cap is attached ( $\mathrm{Gu}$ et al. 2004; Liu et al. 2004). DcpS demonstrates 2500-fold greater activity on a capped dinucleotide versus a full-length capped mRNA substrate, and, moreover, the robust activity of DcpS on capped decay intermediates effectively competes for, and hydrolyzes, cap structure in the presence of the cap-binding protein, eIF4E. These findings imply that DcpS activity will rarely act upon and initiate the decay of fulllength capped mRNAs. Furthermore, DcpS activity can alleviate the potential titration of eIF4E on mRNA oligoribonucleotides unsuitable for translation. Kiledjian also showed that in HeLa cells, mammalian DcpS is predominantly nuclear, but is also present in the cytoplasm distinct from the foci harboring human Dcp2 (Liu et al. 2004). These data suggest that DcpS may play a catalytic and/or regulatory role in the nucleus, and that the two decapping pathways are likely to occur in different subcellular locations in the cytoplasm.

As important as the $5^{\prime}$ end of an mRNA is to its stability, the $3^{\prime}$ terminus also plays a vital role in regulating decay through the presence or absence of polyadenylate residues. Marv Wickens (Univ. of Wisconsin, Madison) introduced the Caenorhabditis elegans GLD2 protein as a divergent member of the DNA polymerase $\beta$ nucleotidyl transferase superfamily that demonstrates poly(A)-addition activity (Wang et al. 2002b). In contrast to nuclear poly(A) polymerases, GLD2 is cytoplasmic and heterodimeric, comprised of one catalytic subunit and one RNA-binding protein subunit. In the absence of the $\mathrm{KH}$ protein, GLD3, for example, GLD2 demonstrates little poly(A) polymerase activity, suggesting that the modular nature of the complex serves to both stimulate polymerase activity and provide substrate specificity through interaction with various subsets of mRNAs. GLD2 represents a founding member of this new class of poly(A) polymerases that also contains, among others, the yeast Trf4 protein (Kwak et al. 2004, and references within).

Although it is generally accepted that polyadenylation of eukaryotic mRNA serves to stabilize mRNA, the role of polyadenylation in the stability of stable, structured RNA has been relatively unexplored. James Anderson (Marquette) discussed the finding that polyadenylation of aberrant tRNA in yeast is destabilizing, akin to the surveillance pathway for defective tRNA and other stable RNAs in E. coli (Li et al. 1998, 2002; Deutscher 2003). Anderson showed that polyadenylation of hypomodified initiator $\mathrm{tRNA}^{\text {met }}$ in the nucleus by Trf4p generates a substrate for exosomemediated degradation of the aberrant species (Kadaba et al. 2004). Interestingly, Trf4p-mediated destabilization is specific for aberrant over wild-type tRNA substrates. Although the nature of this specificity is undetermined, abnormal tRNA structure and/or retention of the aberrant species in the nucleus might offer an explanation for this observation. Phil Mitchell (David Tollervey's laboratory, Welcome Trust Centre, Univ. of Edinburgh) reported that a complex containing Trf4p stimulated the in vitro activity of the exosome on structured RNA substrates. Importantly, multiple rounds of polyadenylation appeared to be required for complete substrate decay, reminiscent of the action of E. coli poly(A) polymerase I (PAP I) in the degradation of structured RNA substrates by the exonucleases, RNase II, and polynucleotide phosphorylase (PNPase) (Coburn and Mackie 1996, 1998).

In bacteria, the destabilizing effect of polyadenylation on structured RNA is due to the creation of a single-stranded RNA "toehold" that provokes exonucleolytic digestion of the substrate by various $3{ }^{\prime}-5^{\prime}$ exonucleases that are otherwise resistant to RNA secondary structure (see Dreyfus and Régnier 2002). It has been unclear, however, how PAP I is targeted to its RNA substrate, prior to poly(A) addition. Work described by Sidney Kushner (Univ. of Georgia) and Philippe Régnier (IBPC, Paris) suggests that in E. coli, the rho-independent transcriptional terminators that exist at the $3^{\prime}$ termini of many mRNAs serve as specific polyadenylation signals for PAP I, and that binding of the Smrelated protein, $\mathrm{Hfq}$, to the terminator modifies this substrate specificity. In support of this concept, Kushner showed that in an $h f q-1$ strain, poly(A) tails are not detectable after rho-independent terminators (Mohanty and Kushner 2004). Under these conditions, PNPase represents the primary poly(A)/polynucleotide-adding activity in the cell, producing short heteropolymeric additions on RNA decay intermediates. Rho-independent terminators, conversely, are poor substrates for polyadenylation by PNPase. Therefore, in a manner resembling the action of GLD3 in $C$. elegans, Hfq binding provides substrate specificity and enhanced activity for polyadenylation catalyzed by PAP I in $E$. coli. Results described by Régnier also suggests that $\mathrm{Hfq}$ alters the recognition of RNA by PAP I and activates poly(A) synthesis. However, he concludes that stimulation of poly(A) addition to mRNAs devoid of $3^{\prime}$ terminal stable secondary structures by $\mathrm{Hfq}$ explains why the fraction of $r p s O$ mRNA polyadenylated downstream of the terminator hairpin is more abundant when $\mathrm{Hfq}$ is inactive (Le Derout et al. 2003). Interestingly, Régnier also demonstrated that, in vitro, Hfq binding to poly (A) tails protects the $3^{\prime}$ terminus from exonucleolytic degradation by RNase II and PNPase (Folichon et al. 2003). Together, these findings present a paradoxical function for $\mathrm{Hfq}$ in the degradation of mRNA 3' ends that requires further analysis.

To further illustrate the bifunctional nature of a poly $(\mathrm{A})$ tail on RNA stability, Laurie Read (SUNY, Buffalo) described opposing effects for poly(A) tail addition on mitochondrial RNA stability in the parasitic protozoan Trypanosoma brucei (Ryan et al. 2003). Read showed that, in vitro, polyadenylation serves to stabilize mitochondrial RNAs that have undergone partial or full editing, while, in contrast, a 
poly(A) tail on an unedited RNA is a signal for rapid turnover. These findings suggest multiple levels of control of mitochondrial gene expression by polyadenylation in trypanosomes.

\section{RIBONUCLEASES AND HELICASES GOVERN MRNA STABILITY}

Ribonucleases represent the most direct trans-acting factors governing mRNA stability in both eukaryotes and prokaryotes. In work presented by Murray Deutscher (Univ. of Miami), the $3^{\prime}$ exonuclease, ribonuclease $\mathrm{R}$, can now be added to the growing list of enzymatic activities that aid in the decay of both structured mRNAs (e.g., repeated extragenic palindrome [REP] sequences) and aberrant stable RNA (e.g., defective tRNA precursors) in E. coli. In contrast to PNPase or RNase II, RNase R efficiently digests through stable, double-stranded RNA helices in vitro, once provided with a single-stranded toehold. Furthermore, this activity does not require ATP or additional factors, such as an RNA helicase, suggesting that RNase R might have the capacity to use energy derived from phosphodiester bond hydrolysis to break intermolecular hydrogen bonds of RNA helices. In vivo, deletion of $r n r$, coupled with a conditional mutant allele for the 3' exonuclease PNPase, causes cell lethality at nonpermissive temperatures and results in the stabilization of small rRNA fragments (Cheng and Deutscher 2003). Titration of ribosomal proteins by the stabilized rRNA fragments could be envisioned to compromise ribosome biogenesis and contribute to the observed cell lethality. RNase $\mathrm{R}$ was also implicated by A.J. Carpousis (CNRS, Toulouse) in a PAP I-dependent pathway of decay for REP sequences in $E$. coli, and, consistent with its function in degrading structured RNA, Philippe Régnier described a role for RNase $\mathrm{R}$ in the poly(A)-dependent degradation of $r p s O$ mRNA protected by the stable hairpin of its rho-independent terminator.

In yeast, RNA cleavage by the RNase III homolog, Rntlp, requires a double-stranded RNA stem-loop structure capped by an AGNN-type tetraloop (Wu et al. 2001). Using whole genome theoretical folding and gene expression profiling in $r n t 1 \Delta$ mutant cells, Guillaume Chanfreau (UCLA) described the identification of numerous mRNA substrates for Rnt1p. In addition to playing a role in the regulation and degradation of unspliced pre-mRNAs coding for ribosomal proteins (Danin-Kreiselman et al. 2003), Rntlp was shown to control the decay of several genes involved in the response to iron starvation. Consistent with a role for Rntlp in anti-iron toxicity, $r n t 1 \Delta$ strains exhibit sensitivity to high concentrations of iron. Further characterization of Rntlp by Sherif Abou-Elela (Univ. of Sherbrook) demonstrated that, in vitro, Rntlp can bind not only to its characterized dsRNA substrate, but also to dsDNA (Lamontagne et al. 2004). Although cleavage of dsDNA by Rntlp was not observed in vitro, Rntlp was shown to cleave
DNA/RNA hybrids in yeast extracts, independent of ATP. These data suggest Rnt1p can exhibit RNA-dependent deoxyribonuclease activity, a finding that might spur new antiviral strategies.

In E. coli the decay of many, if not most, mRNAs is initiated by an endonucleolytic cleavage catalyzed by RNase E (Kushner 2002). In vertebrates, however, few endonucleases have been identified and, in most cases, their role in initiating RNA decay is unknown (Schoenberg and Chernokalskaya 1997). Notwithstanding, the best characterized protein within this class of enzymes is PMR1, a polysomeassociated endonuclease that selectively degrades serum protein mRNAs in Xenopus liver following estrogen stimulation (Pastori et al. 1991a,b). It has remained unclear, however, how PMR1 is targeted and restricted to specific mRNA substrates. To facilitate the characterization of PMR1 targeting, Daniel Schoenberg (Ohio State Univ.) described a Cos-1 cell system that accurately recapitulates PMR1-mediated mRNA decay. Using this system, protein domains important for targeting of PMR1 to polysomes were shown to be necessary for the association of PMR1 with its substrate mRNA (i.e., albumin mRNA), and, in agreement with this, albumin mRNA is stabilized in PMR1 polysome targeting mutants. Furthermore, target mRNA blocked for translation does not become associated with PMR1 and is prevented from being rapidly degraded. These findings suggest a model whereby PMR1 is recruited to its polysomeassociated target through interaction with an RNP specific to the mRNA (Yang and Schoenberg 2004). Consistent with an association between PMR1 and actively translating mRNA, PMR1 is found distributed throughout the cytoplasm and does not colocalize with human Dcp1 protein in Cos-1 cells (Yang and Schoenberg 2004).

Of significant interest to those in the field of RNA interference (RNAi) is the isolation and characterization of the enzymatic activity that mediates siRNA interaction with its cognate target and subsequent mRNA cleavage. Eric Sontheimer (Northwestern) described the use of native gel electrophoresis to analyze complexes from Drosophila that mediate RNAi. Exogenous short interfering RNAs (siRNAs) were shown to initially associate with the RNase III homo$\log$, Dicer-2, in an RNAi initiator complex that cofractionates with dsRNA processing activity. The initiator complex acts as a precursor to a very large $(\sim 80 S)$ RISC effector complex that also contains Dicer-2 and siRNA, indicating that Dicer-2 does not pass newly processed siRNAs to a distinct complex, but rather plays a role beyond the initiation phase of RNAi. The $80 \mathrm{~S}$ complex can bind and cleave targeted mRNAs in a cognate siRNA-dependent manner, and is being considered a holoRISC/RNAi effector complex (Pham et al. 2004). Frédéric Souret (Pamela Green's laboratory, Univ. of Delaware) showed that in Arabidopsis, 3' products of miRNA-mediated mRNA cleavage can be substrates for XRN4, one of three plant homologs of the yeast Xrn1 protein (Souret et al. 2004). The cytoplasmically lo- 
calized AtXRN4 plays a clear role in the stability of $3^{\prime}$ cleavage products generated by the miRNA-directed cleavage of several mRNA targets, but, curiously, it manifests no growth phenotype when deleted in plants. This work implicates an Xrn1 homolog in mRNA degradation in a multicellular eukaryote, and denotes a new role for the $5^{\prime}-3^{\prime}$ exonuclease in the miRNA-mediated pathway of mRNA decay.

The role for RNA helicases as trans-acting regulators in multiple RNA-related processes, including mRNA turnover, is critical. For example, A.J. Carpousis provided the first evidence that the nonessential E. coli helicase, RhlB, is required for mRNA decay in vivo (Khemici and Carpousis 2004). RhlB was shown to enable the degradation of highly structured REP sequences by PNPase. Interestingly, this activity requires the association of both proteins with RNase $\mathrm{E}$ as components of the RNA degradosome. Therefore, in addition to initiating mRNA turnover through endonucleolytic cleavage, the multienzyme degradosome complex is also involved in the $3^{\prime}-5^{\prime}$ decay of structured mRNA fragments. Work was also presented by Carpousis suggesting that RhlB plays a role in initiating mRNA degradation through facilitating endonucleolytic cleavage by RNase E, presumably by unwinding RNA structure to expose cleavage sites. Two additional E. coli DEAD-box RNA helicases, SrmB and CsdA, were shown by Marc Dreyfus (CNRS, Paris) to be involved in assembly of the $50 \mathrm{~S}$ ribosomal subunit (Charollais et al. 2003, 2004). In vitro experiments assessing the substrate specificity and mechanisms of the helicases indicate that they unwind duplexes with either $5^{\prime}$ or $3^{\prime}$ single-stranded RNA extensions, but that they demonstrate low processivity. An interpretation of this work is that helicases work in a highly ATP-consuming, direct pulling apart of the RNA strands, and not in an inch-worm type mechanism as previously hypothesized (Bizebard et al. 2004).

\section{TRANS-ACTING FACTORS MODULATE THE STABILITY OF MRNA SUBPOPULATIONS}

Some trans-acting factors demonstrate regulatory specificity in cis by interacting with and modulating the stability of a subset of mRNAs, while, conversely, others network directly with the ribonucleases that mediate mRNA degradation. In an example of the latter, the E. coli protein RraA was described by Stanley Cohen (Stanford) to interact with and inhibit RNase E endoribonuclease activity (Lee et al. 2003; Monzingo et al. 2003). RraA does not interact detectably with RNA substrates, but was shown by microarray profiling of $r a A \Delta$ cells to modulate the stability of numerous mRNAs $(\sim 80)$ at least twofold (Lee et al. 2003). Interestingly, inhibition of RNase E activity by RraA does not require the RNase E carboxy-terminal scaffold region important in formation of the RNA degradosome; however, RraA binding is enhanced by the carboxy-terminal half of RNase
E. Cohen also presented evidence for a second regulatory protein, RraB, which also modulates the catalytic function of RNase E.

In eukaryotes, PUF proteins interact directly with specific RNA targets through sequence motifs within the $3^{\prime}$ UTR to regulate both translation status and mRNA stability (for review, see Wickens et al. 2002). Findings were presented by two speakers describing progress in characterizing the interaction between the conserved structural motifs of the PUF protein family and the specific RNA sequences found within target mRNAs. Marv Wickens discussed the elucidation of consensus sequences for the binding of several yeast PUF proteins. Additionally, the specificity of RNA binding was shown to be inherent in the structure of the conserved PUF repeat domains as demonstrated by the hybrid binding specificity of chimeric proteins generated by swapping of PUF repeat elements between two C. elegans PUF proteins. Wendy Olivas (Univ. of Missouri, St. Louis) furthered this discussion by detailing both the identification of Puf3p consensus binding sites on COX17 mRNA (Jackson et al. 2004) and, more appreciably, the identification and confirmation of mRNA targets for the previously uncharacterized Pufl and 2 proteins of yeast. An exciting outcome of her work was the demonstration that Puf proteins in yeast can either stimulate mRNA decay (through accelerated deadenylation and/or decapping) or stabilize target mRNAs.

AU-rich element (ARE)-mediated decay represents a classic example of how trans-acting regulators influence degradation of a subset of mRNAs (for review, see Brennan and Steitz 2001). Perry Blackshear (NIEHS, North Carolina) described the deletion of the ARE-binding protein, tristetraprolin (TTP), in mice. TTP-deficient mice develop a systemic inflammatory syndrome as a consequence of enhanced secretion of TNF $\alpha$ and GM-CSF, the products of two ARE-containing mRNAs. TTP has previously been shown to stimulate the deadenylation of these messages (Lai et al. 2003), and consistent with this, it was shown that the enhanced secretion of TNF $\alpha$ in the mice was due to stabilization of its mRNA. Interestingly, deletion of a different TTP-family member (i.e., Zfp36L1/CMG-1) resulted in chorioallantoic fusion defects and embryonic lethality in the mice (Stumpo et al. 2004). The distinct phenotypes observed for the knock-out mice highlights that these two homologous proteins have distinct subsets of RNA substrates, and that in the case of Zfp36L1, at least one of these substrates is critical for proper embryonic development. Following up on this concept, Heidy Hau (Paul Bohjanen's laboratory, Univ. of Minnesota) showed that different ARE sequences exhibit different affinities for TTP and HuR, an ARE-binding protein whose interaction with mRNA results in stabilization rather than destabilization (Raghavan et al. 2001). Importantly, the relative binding affinities of these proteins to the AREs were shown to correlate with transcript fate. Since both HuR and TTP are induced upon 
stimulation of $\mathrm{T}$ cells, these findings suggest that the RNAbinding affinities, relative abundances, and the opposing effects of these proteins on mRNA stability all contribute to differential degradation rates of ARE-containing mRNAs following $\mathrm{T}$ cell activation.

Paul Anderson (Harvard Medical School) added to the complexity of ARE-mediated mRNA decay by showing that phosphorylation of TTP by MAPKAP kinase-2 (MK2) upon $T$ cell activation serves to abrogate TTP-dependent degradation of ARE-containing mRNA (Stoecklin et al. 2004). Moreover, inhibition of TTP activity upon phosphorylation was determined to result from binding of TTP to the regulatory adaptor protein 14-3-3. Interestingly, TTP:14-3-3 complex formation excludes TTP from stress granules (SGs), the dynamic cytoplasmic foci which harbor stalled translation initiation complexes in conditions of environmental stress (see Kedersha and Anderson 2002), suggesting that the decision to degrade ARE-containing RNA may occur, to some degree, in SGs (Anderson et al. 2004; Stoecklin et al. 2004). Of note is the observation that under conditions of stress, SGs juxtapose with P-bodies, the cytoplasmic foci where mRNA decay can occur (Sheth and Parker 2003; Cougot et al. 2004), and, moreover, overexpressed levels of TTP are found colocalized with P-bodies (Stoecklin et al. 2004). The relationship between TTP-mediated mRNA decay, the recycling of translational arrested mRNA complexes from SGs, and localized mRNA turnover in P-bodies is fascinating, and will certainly be the focus of further experimentation.

In a manner reminiscent of the ARE-binding proteins, the Sm-like eubacterial RNA chaperone, Hfa, plays a role in RNA stabilization through the binding of AU-rich, singlestranded RNA in bacteria. Udo Blasi (Univ. of Vienna) has previously shown that $\mathrm{Hfq}$ can mediate the rapid degradation of the E. coli ompA mRNA by binding to sequences in the $5^{\prime}$ UTR, leading to structural rearrangements involving the ribosome binding site (Vytvytska et al. 2000). Here, Blasi showed that Hfq plays a protective role through binding of AU-rich sequences within the RyhB and DsrA small regulatory RNAs (sRNAs) which overlap with RNase E recognition sites (Moll et al. 2003). The stabilization of sRNAs by Hfq further influences mRNA stability through modulating sRNA availability to its cognate mRNA target. For example, stabilization of RyhB by Hfq leads to increased interaction of the sRNA with its target, $s o d B$ mRNA, that results in functional inactivation and enhanced decay of the mRNA (Vecerek et al. 2003). Interestingly, Hfq levels are growth-rate controlled and are in abundance in E. coli during slow growth (Vytvytska et al. 1998), suggesting that the regulation of mRNA levels of the primary and/or secondary RNA targets of Hfq is important for adaptation to limiting growth conditions.

In Arabidopsis, microarray experiments indicate that select mRNAs containing the cis-acting "downstream" (DST) element (Perez-Amador et al. 2001) or mRNAs identified as unstable (Gutierrez et al. 2002) are regulated temporally by the circadian clock. In her presentation, Pam Green (Univ. of Delaware) established that circadian regulation efficiently controls the differential stability of certain mRNAs harboring the DST instability determinant at different times throughout the day (Gutierrez et al. 2002). Consistent with this, $d s t 1$ mutants are deficient in circadian clock-regulated decay of CCL mRNA (a unique subclass of DST-containing mRNA) and, interestingly, are also defective in circadianregulated leaf movement.

In some situations, the RNA targets of trans-acting regulators of decay are not manifested as simple primary sequence recognition elements. Such is the case for mRNA substrates of the trans-acting regulator of nonsense-mediated decay (NMD), UPF1. In an effort to identify natural mRNA substrates of NMD, Hal Dietz (HHMI, Johns Hopkins) and Andreas Kulozik (Univ. of Heidelberg) presented microarray data from human cell lines depleted of UPF1 (hUPF1). Expression profiles from the Dietz laboratory revealed the upregulation of various subsets of RNAs, which, not unexpectedly, all manifest a spliced intron downstream of the termination codon. This architecture is consistent with the proposed role of an exon junction complex (EJC) in promoting NMD through an interaction with Upf proteins (see Singh and Lykke-Andersen 2003). These studies also identified an increase in expression of genes involved in amino acid metabolism and transport, suggesting that under conditions of amino acid starvation, inhibition of translation and, consequently, inhibition of NMD preferentially increases the expression of mRNAs that promote restoration of amino acid homeostasis. Dietz also discussed that while UPF1 is essential for embryonic viability in mice (Medghalchi et al. 2001), mice expressing a dominant-negative UPF1 allele are viable, and show impaired T-cell maturation, which correlates temporally with the stabilization of nonsense-containing TCR $\beta$ mRNA generated by nonproductive rearrangements. Together these findings suggest that the NMD pathway may act as a molecular capacitor by restraining phenotypic variation under normal conditions and releasing this variation when functionally compromised.

The present model for NMD in mammalian cells suggests a linear recruitment of UPF3, UPF2, and UPF1 to the RNA by EJC components (see Singh and Lykke-Andersen 2003). It was, therefore, anticipated that RNA substrates which increased in abundance in hUPF1-depleted cells would also show increased expression in hUPF2- and hUPF3b-ablated cells. Kulozik presented data showing, in contrast, that only a subset of the hUPF1-modulated mRNAs respond to the depletion of hUPF2 (while the data gathered from the deletion of hUPF3b was inconclusive). These findings are in opposition to data presented by Dietz showing that transcripts regulated by UPF1 are also regulated by UPF2 and UPF3. It is noteworthy that both groups have analyzed only a small number of UPF1 targets and, therefore, further 
studies will be required to understand the basis for this contradictory observation. Kulozic further discussed experiments in which various NMD factors were tethered to the $3^{\prime}$ UTR of otherwise normal mRNAs in depleted cells. These studies indicate that hUPF1 represents a late step in the NMD pathway, but that NMD substrates exhibit differential requirements for hUPF2 and hUPF3b and components of the exon junction complex such as Y14/MAGOH, eIF4AIII, or RNPS1. These findings challenge the model of a single, linear NMD pathway as previously put forward; however, they should be interpreted with caution as they are based on a tethering assay that may bypass some essential steps of the NMD pathway.

\section{ALTERNATIVE FATES FOR mRNAs HARBORING STALLED OR ABERRANTLY TERMINATING RIBOSOMES}

A delicate relationship exists between mRNA translation and stability in both eubacteria and eukaryotes. Specifically, mRNAs that are functional and translated efficiently are generally stabilized, while mRNAs that are either aberrant or whose protein products are no longer required are rapidly degraded. In eukaryotes, this relationship can be exemplified by the nonsense-mediated mRNA decay pathway, in which mRNA harboring a premature termination codon (PTC) is subject to rapid mRNA degradation by deadenylation-independent mRNA decapping and $5^{\prime}-3^{\prime}$ exonucleolytic degradation, and/or the $3^{\prime}-5^{\prime}$ pathway of decay (for review, see Baker and Parker 2004; Maquat 2004). One important question in understanding the mechanism of NMD is the nature by which a PTC is recognized as aberrant. Allan Jacobson (Univ. of Massachusetts Medical School) began to address this question using a toeprint assay to demonstrate that, in vitro, translation termination at a PTC is distinct from termination at a normal stop codon. In contrast to normal termination, ribosomes at a PTC fail to efficiently release and have a propensity to migrate to upstream AUG sequences. The aberrant ribosome toeprint at a PTC was shown to require Upf1p, and, interestingly, was eliminated by either flanking the PTC with the native 3' UTR or tethering Pablp downstream of the PTC. Thus, in yeast, activation of NMD appears to be directly linked to the intrinsically aberrant nature of premature termination. Moreover, the requirement for a "normal" relationship between the terminating ribosome and a properly configured mRNA 3' UTR supports the "faux UTR" model for NMD in yeast (Hilleren and Parker 1999; Jacobson and Peltz 2000).

In her presentation, Elisa Izaurralde (EMBL, Germany) showed that, in Drosophila, aberrant ribosome termination at a PTC leads to endonucleolytic cleavage of the NMD substrate in the vicinity of the termination event (Gatfield and Izaurralde 2004). Strikingly, initiation of decay of an NMD substrate by endonucleolytic cleavage has not been previously described and has, to date, been observed only in Drosophila. This pathway prevails in flies despite the conservation of NMD machinery components between flies, worms, and humans, and mechanistic commonalities shared between flies and yeast (Gatfield et al. 2003). Although considerable effort has been given to identifying the activity responsible for the endonucleolytic cleavage event, the activity has yet to be defined. In observations similar to those previously made in yeast, NMD in flies exhibits a polarity effect whereby $5^{\prime}$-proximal PTCs elicit a stronger NMD response than those distal to the $5^{\prime}$ end of the mRNA. In yeast, the length of $3^{\prime}$ UTR elements is reasonably invariable, and the polarity effect has been considered in terms of the relative distance between the PTC and 3' UTR elements (Cao and Parker 2003). However, it was noted that in Drosophila, the $3^{\prime}$ UTRs of mRNAs are significantly diverse in size such that a model simply hinging upon the relative distance between a terminating ribosome and elements within the $3^{\prime}$ UTR to explain the strength of an NMD response is unlikely.

Such a relationship, in contrast, appears to be important in the cell-cycle-regulated stability of histone mRNA. In metazoa, histone mRNAs are not polyadenylated and bound by poly(A) binding protein, but, rather, are stabilized by a $3^{\prime}$ terminal stem-loop structure that is bound by stem-loop binding protein (SLBP) (for review, see Marzluff and Duronio 2002). Bill Marzluff (Univ. of North Carolina, Chapel Hill) indicated that the position of the $3^{\prime}$ stem-loop must be 25-50 nt from the stop codon to participate in regulated histone mRNA stability, highlighting the importance of a proper spatial relationship between a ribosome engaged in translational termination and the stem-loop/ SLBP complex. Marzluff also demonstrated that regulated histone mRNA stability was affected by depletion of hUPF1, and, consistent with this, inhibition of hUPF1 phosphorylation by the Smg1 homolog, ATR, led to stabilization of histone mRNA. How UPF1 modulates the relationship between "normal" translation termination and histone mRNA $3^{\prime}$ stem-loop elements in a cell-cycle-dependent manner is presently unknown, but may provide important insight into the mechanism of NMD in mammalian cells.

In bacteria, mRNAs harboring a stalled ribosome can undergo two unique fates. In the first case, Hiroji Aiba (Nagoya Univ., Japan) and Christopher Hayes (Bob Sauer's laboratory, MIT) reported that, dependent upon the codon context, ribosomes engaged in normal translation in E. coli can stall during elongation, and that pausing at either a sense or a stop codon results in mRNA cleavage that generates termini ending either within or immediately adjacent to the pause site (Hayes and Sauer 2003; Sunohara et al. 2004a,b). Significantly, the carboxy-terminal amino acids of the nascent polypeptide chain, frequently arginine and proline, were implicated in enhancing the ribosome pausing through their conformational and/or chemical properties (Sunohara et al. 2002; Hayes et al. 2002a,b). Interestingly, 
the $5^{\prime}$ product of the mRNA cleavage event was shown to be a substrate for trans-translation by the tmRNA quality control system (for review, see Withey and Friedman 2003), suggesting that the stalled ribosome remains bound to the 5 ' cleavage product, and its release is facilitated by tmRNA. It was proposed that the coupling of ribosome arrest with mRNA cleavage and trans-translation ensures quality control of protein synthesis and may represent a mechanism of regulating expression of particular genes. Analyses of the mRNA products generated after ribosome stalling indicate that they are created from an endonucleolytic cleavage event (Sunohara et al. 2004a,b). Markedly, cleavage does not require tmRNA, bacterial toxins such as RelE (see below), or any other known endoribonuclease in E. coli.

In the second case, mRNAs harboring stalled ribosomes are substrates for endonucleolytic cleavage in the ribosomal A-site promoted by the toxin RelE (Pedersen et al. 2003). Ken Gerdes (Univ. of Southern Denmark, Odense) explained that in E. coli, mRNA cleavage involving RelE occurs predominantly under conditions of nutritional stress and leads to global inhibition of protein synthesis. Gerdes also proposed that RelBE and similar antitoxin:toxin systems serve to facilitate recovery from limiting growth conditions and represent conserved stress response elements (Christensen et al. 2003). Consistent with this viewpoint, the toxic affect of RelE activity is counteracted by tmRNA, indicating that the $5^{\prime}$ product of mRNA cleavage is also a substrate for trans-translation and that rapid ribosome recycling and amino acid salvage is critical for cell survival (Christensen and Gerdes 2003). Curiously, in vitro, RelE does not demonstrate ribonuclease activity on mRNA free of ribosomes, but, rather, cleaves only mRNA bound by stalled ribosomes (Pedersen et al. 2003). These observations suggest that RelE either acquires RNase activity upon ribosome binding or it triggers RNA cleavage by an as yet unidentified ribonuclease. In light of the fate of the mRNA in both this and the above situations, it was not overlooked at this meeting that, when stalled on an mRNA, the ribosome itself may catalyze the endonucleolytic cleavage event (Christensen and Gerdes 2003; Hayes and Sauer 2003; Sunohara et al. 2004a).

In eukaryotes, mRNAs devoid of an in-frame translational termination codon permit ribosome translocation to proceed to the extreme $3^{\prime}$ terminus and are the targets for rapid degradation by the nonstop mRNA decay pathway (Frischmeyer et al. 2002; van Hoof et al. 2002). It is hypothesized that, similar to truncated mRNA in bacteria, nonstop mRNAs harbor ribosomes stalled at the $3^{\prime}$ termini that are required to be removed prior to decay of the transcript. A model for nonstop decay was presented by Ambro van Hoof (Univ. of Texas, Houston) proposing that, in yeast, the Ski7 protein interacts with the A-site of a ribosome stalled at the end of a nonstop mRNA and facilitates its release from the mRNA. Furthermore, the interaction between Ski7p and the stalled ribosome was suggested to promote decay of the mRNA through recruitment of the exosome complex. In support of this model, the carboxyterminal domain of Ski7p, which shows homology to the GTPase eRF3, was shown to be required for nonstop decay, but not for normal mRNA turnover. Furthermore, the $\mathrm{N}$ terminus of Ski7p, which interacts indirectly with the exosome complex, is required to be in cis with the carboxyterminal domain to mediate mRNA degradation. Therefore, in nonstop decay, Ski7p may act both as a release factor mimic to liberate stalled ribosomes and as a recruiter for the $3^{\prime}-5^{\prime}$ mRNA decay machinery.

Recognition and aberrant translation termination at a PTC is conventionally believed to occur by ribosomes engaged in translation in the cytoplasm. However, some PTCcontaining messages manifest reduced levels in the nuclear fraction of cells (e.g., Belgrader et al. 1993), and PTC recognition has been reported to cause alterations in events that clearly occur in the nucleus. For example, a PTC at various positions within the immunoglobulin $\mu(\operatorname{Ig}-\mu)$ gene results in the accumulation of its pre-mRNA in the nucleus near the site of transcription (Mühlemann et al. 2001). Likewise, PTCs upregulate the level of TCR $\beta$ pre-mRNA at or near the site of transcription (Mühlemann et al. 2001). Miles Wilkinson (Univ. of Texas) provided additional evidence in support of this nonsense-mediated upregulation of pre-mRNA (NMUP) response. He demonstrated that the target of NMUP is not unspliced TCR $\beta$ mRNA but rather a partially spliced species containing two introns. Furthermore, mapping experiments revealed that the TCR $\beta$ VDJ exon and adjacent intron sequences are sufficient to elicit NMUP. Wilkinson also discussed an additional intranuclear response to a PTC referred to as nonsense-associated altered splicing (NAS), in which alternatively spliced mRNA void of the offending nonsense mutation is upregulated. While many cases of NAS are the result of nonsense mutations disrupting splicing enhancers (see Maquat 2002), in the case of the TCR $\beta$ gene, nonsense and frameshift mutations increase alternatively spliced mRNA due to disruption of reading frame (Wang et al. 2002a). Wilkinson presented preliminary evidence that the TCR $\beta$-specific NAS response is the result of a feedback mechanism that increases the production of alternatively spliced mRNA in response to a PTC detected in constitutively spliced mRNA.

Oliver Mühlemann (Univ. of Bern, Switzerland) described yet another intranuclear response to a nonsense codon: nonsense-mediated transcriptional gene silencing (NMTGS). He reported that introduction of a PTC in an Ig- $\mu$ gene causes reduced transcription, as assessed by nuclear run-on analysis. While NMTGS is seemingly contradictory to the NMUP response, as the former decreases pre-mRNA levels and the latter increases pre-mRNA levels, the observation might be reconciled by the fact that they occur in different circumstances, NMTGS in transfected HeLa cells and NMUP in B-cell hybridomas. The mechanism of NMTGS is not yet clear; nonetheless, it appears to 
involve the formation of heterochromatin (as determined by histone acetylation/methylation status), suggesting that chromatin remodeling may play a role in mediating this form of gene silencing. Accordingly, Mühlemann also presented preliminary evidence that small dsRNAs and the RNAi pathway may be involved in mediating NMTGS, similar to the heterochromatic silencing by RNAi examined in fission yeast (Volpe et al. 2002) and in plants (Zilberman et al. 2003).

Two models have been put forward to explain how a PTC, whose recognition requires ribosome scanning, might impact events in the nucleus (Wilkinson and Shyu 2002; Dahlberg et al. 2003; Dahlberg and Lund 2004). One involves an undefined "feedback mechanism" whereby the detection of aberrant mRNA by cytoplasmic ribosomes signals changes in gene expression patterns in the nucleus. The second model pivots around the controversial yet unresolved issue of nuclear translation. Only future experimentation will help distinguish which of the models, if either, contributes to PTC-induced intranuclear events.

\section{STRUCTURAL DATA PROVIDE INSIGHT INTO MRNA TURNOVER}

Protein structure elucidation is beginning to play an important role in understanding both the detailed catalytic events performed by ribonucleases and the protein-protein or protein-RNA interactions that act to regulate catalytic activity. Haiwei Song (Institute of Molecular and Cell Biology, Singapore) discussed the elucidation of the crystal structures of the yeast Dcp1 and Ski8 proteins and their implications for mRNA turnover (Cheng et al. 2004; She et al. 2004). In particular, mutation of conserved sequences determined to be on the molecular surface of Dcplp has facilitated the identification of residues critical for mediating interactions with regulatory proteins involved in mRNA decapping (She et al. 2004). In separate presentations, the crystal structures of the E. coli DEDD exonucleases, RNase $\mathrm{D}$ and oligoribonuclease (Fiedler et al. 2004), were described by Yuhong Zuo from Arun Malhotra's laboratory (Univ. of Miami). While these proteins share similar sequence motifs at the amino acid level, structural analysis indicates that they exhibit strikingly dissimilar structural domains. These two homologous proteins exhibit strong differences in both RNA substrate preference and catalytic function, and it is anticipated that comparison between these and other DEDD protein structures will assist in understanding these functional divergencies.

Kenneth McDowall (Univ. of Leeds) reported a collaboration with the group of Ben Luisi (Cambridge) in which biophysical techniques were being used to examine the role of metal in determining the structure and function of RNase E. It was shown that an intramolecular pairing of amino acid residues within a highly conserved motif serves to coordinate zinc as part of a dimerization interface. Importantly, mutation of conserved cysteine residues comprising the zinc coordination site results in disruption of the RNase
E tetramer into stable dimmers, which maintain RNA binding capacity but show little catalytic activity. Moreover, tetrameric RNase E, formed by chemical crosslinking in the absence of zinc, was found to be catalytically active. These results suggest that metal ion binding by RNase E enhances enzyme activity by promoting tetramerization rather than increasing RNA binding or activating catalysis directly.

John McCarthy (Univ. of Manchester, UK) reported on NMR and cryo-EM studies aimed at understanding the mechanisms underlying stabilization of the eIF4E:cap interaction by eIF4G and ribosome recruitment to the mRNA $5^{\prime}$ end during cap-dependent translational initiation in eukaryotes (von der Haar et al. 2004). Significantly, eIF4G interaction with cap-bound eIF4E elicits a coupled folding transition in which eIF4G wraps around the loosely structured N-terminal domain of eIF4E, which, in turn, is coupled to conformational changes at distal locations of eIF4E that are presumably responsible for increased cap affinity (Gross et al. 2003). McCarthy also described a dramatic conformational change that befalls the $40 \mathrm{~S}$ ribosomal subunit upon binding of the initiation factors that prepare this subunit for recruitment to the $5^{\prime}$ end of the mRNA. This structural alteration can be envisioned as a switch promoting an association between the $43 \mathrm{~S}$ ribosome complex and the mRNA which renders the complex competent for scanning along the $5^{\prime}$ UTR. In light of the intimate relationship between translation initiation and mRNA decapping (see Coller and Parker 2004), a more detailed understanding of the steps involved in initiation factor and $43 \mathrm{~S}$ ribosomal subunit binding to the mRNA $5^{\prime}$ cap will undoubtedly offer insight into the transition between a translationally competent mRNP and one destined for degradation.

Elena Conti (EMBL, Germany) presented the crystal structure of the amino-terminal domain of human SMG7. SMG7 is one of three related and essential NMD-associated proteins (i.e., SMG5, 6, and 7) which, in metazoans, targets phosphorylated UPF1 for dephosphorylation by protein phosphatase 2A (PP2A) (Page et al. 1999; Anders et al. 2003; Chiu et al. 2003). The structure reveals that hSMG7 displays a strong similarity with $14-3-3$, a signal transduction protein that binds serine-phosphorylated proteins (Tzivion and Avruch 2002; Yaffe 2002). The phosphoserine-binding site of 14-3-3 is conserved in hSMG7, suggesting that SMG7 may bind serine-phosphorylated UPF1 via its N-terminal 14-3-3-like domain. Together with data from Elisa Izaurralde showing that hSMG7 localizes to cytoplasmic mRNA decay foci, a picture is emerging where SMG7 provides a link between proteins of the NMD surveillance complex and those of mRNA turnover.

\section{ADDITIONAL MODEL ORGANISMS FOR THE ANALYSIS OF MRNA DECAY}

It is now generally accepted that $E$. coli will provide only a part of the whole eubacterial RNA decay puzzle. Studies in 
Bacillus subtilis, Streptomyces coelicolor, and Synechocystis species, while preliminary, are offering alternative model organisms for the advancement of our understanding of RNA metabolism. Presently, identification of the major enzymatic activities and pathways for mRNA decay is the goal. For example, David Bechhofer (Mount Sinai, New York) reported that in B. subtilis, many of the decay activities characterized in E. coli (e.g., RNase E, RNase II, and oligoribonuclease), are absent, and that PNPase has emerged as one of the key enzymes in mRNA turnover. Expression profiling of a pnp mutant led to the discovery that PNPase is the primary enzyme required for the degradation of many small decay intermediates and that this property is critical both for recycling of the B. subtilis trp operon regulator, TRAP (Deikus et al. 2004), and for growth on ammonium as sole nitrogen source. Interestingly, PNPase was identified as the primary polyadenylation, or more accurately, polynucleotidylation activity in Synechocystis, S. coelicolor, and B. subtilis (Gadi Schuster [Israel Institute of Technology, Israel], Gabriela Cohen [Stanford], and Cinvestav-Unidad Olmedo-Alvarez [Irapuato, Mexico] laboratories, respectively). These discoveries provide a significant step forward toward understanding alternative pathways of mRNA decay in eubacteria.

While the enzymes and pathways of eukaryotic mRNA degradation are best characterized in the unicellular budding yeast, Saccharomyces cerevisiae (see Parker and Song 2004), it is now evident that additional eukaryotic organisms will contribute significantly to furthering our understanding of mRNA turnover. Excitingly, analysis of "alternative" models are both corroborating previous findings and exposing new players and mechanisms for mRNA decay. For example, Elmer Wahle (Univ. of Halle, Germany) reported that the CCR4-NOT complex, the major cytoplasmic mRNA deadenylase in yeast (Tucker et al. 2001, 2002), is conserved in Drosophila. Furthermore, the CCR4 and CAF1 protein homologs were shown to exhibit poly(A)specific $3^{\prime}$ exoribonuclease activity in vitro, while $c c r 4 \mathrm{mu}-$ tant flies manifest elongated bulk poly(A) mRNA (Temme et al. 2004). Meanwhile, the nonsense-mediated pathway of decay in flies appears to be unique (see Izaurralde above). One obvious advantage for studying Drosophila as a model organism is the ability to use genetic techniques such as transfection and efficient RNAi-mediated factor depletion of cultured Drosophila cells coupled with the evaluation of complex physiological phenotypes as a consequence of genetic defects in whole animals.

Additional model organisms each offer unique advantages for the analysis of RNA metabolism in eukaryotes. For example, in Arabidopis, multiple homologs of characterized decay factors are being identified that, at first glance, might be considered redundant nucleases. Yukako Chiba (Pamela Green's laboratory, Univ. of Delaware) described seven homologs of the deadenylase complex protein CCR4p in Arabidopsis. Microarray analyses of plants mutant for two of the members indicate that while the enzymes share some putative mRNA substrates in common, most substrates are unique to each particular enzyme. Based on these findings, analysis of mRNA decay in Arabidopsis may allow for an understanding of small subtleties in nuclease function and substrate specificity as well as uncover regulatory mechanisms that underlie the need for several proteins with similar catalytic activities. Finally, it is undeniable that the advent of RNAi as a reverse genetic technique has made mammalian primary and transformed cells suitable targets for extensive molecular analysis. It can be predicted that it is only a matter of time before this technology provides an important link between our ability to acquire knowledge about RNA metabolism and the implementation of therapeutic strategies that regulate gene expression at the level of RNA.

\section{CONCLUDING REMARKS}

This meeting highlighted the synergy and camaraderie that is openly shared between scientists in the field of eubacterial and eukaryotic mRNA decay. Although it is apparent that no canonical pathway for mRNA degradation exists, the collective advancement in both of these areas of research continues to uncover mechanisms in which RNA is targeted and fed into this important pathway for posttranscriptional regulation of gene expression. Interestingly, since the last gathering of this group of researchers, several descriptions of cytoplasmic foci which harbor various RNA decay proteins have been communicated (van Dijk et al. 2002; Ingelfinger et al. 2002; Sheth and Parker 2003; Cougot et al. 2004). These observations have noticeably impacted on how the field is "looking" at mRNA degradation; however, the full impact of subcellular localization of mRNA degradation remains to be unveiled.

\section{ACKNOWLEDGMENTS}

The authors are regretful to those participants whose work was not cited or discussed due to space limitations. In addition, we are grateful to our colleagues for communicating data prior to publication and to those individuals who made helpful suggestions for the revision of this meeting review.

\section{REFERENCES}

Anders, K.R., Grimson, A., and Anderson, P. 2003. SMG-5, required for $C$. elegans nonsense-mediated mRNA decay, associates with SMG-2 and protein phosphatase 2A. EMBO J. 22: 641-650.

Anderson, P., Phillips, K., Stoecklin, G., and Kedersha, N. 2004. Posttranscriptional regulation of proinflammatory proteins. J. Leukoc. Biol. 76: 42-47.

Baker, K.E. and Parker, R. 2004. Nonsense-mediated mRNA decay: Terminating erroneous gene expression. Curr. Opin. Cell Biol. 16: 293-299.

Belgrader, P., Cheng, J., and Maquat, L.E. 1993. Evidence to implicate 
translation by ribosomes in the mechanism by which nonsense codons reduce the nuclear level of human triosephasphate isomerase. Proc. Natl. Acad. Sci. 90: 482-486.

Bizebard, T., Ferlenghi, I., Iost, I., and Dreyfus, M. 2004. Studies on three E. coli DEAD-box helicases point to an unwinding mechanism different from that of model DNA helicases. Biochemistry 43: 7857-7866.

Brennan, C.M. and Steitz, J.A. 2001. HuR and mRNA stability. Cell. Mol. Life Sci. 58: 266-277.

Cao, D. and Parker, R. 2003. Computational modeling and experimental analysis of nonsense-mediated decay in yeast. Cell 113: 533-545.

Charollais, J., Pfleiger, D., Vinh, J., Dreyfus, M., and Iost, I. 2003. The DEAD-box RNA helicases SrmB is involved in the assembly of $50 \mathrm{~S}$ ribosomal subunits in Escherichia coli. Mol. Microbiol. 48: 12531265.

Charollais, J., Dreyfus, M., and Iost, I. 2004. CsdA, a cold-shock RNA helicases from Escherichia coli, is involved in the biogenesis of $50 \mathrm{~S}$ ribosomal subunit. Nucleic Acids Res. 32: 2751-2759.

Cheng, Z.F. and Deutscher, M.P. 2003. Quality control of ribosomal RNA mediated by polynucleotide phosphorylase and RNase R. Proc. Natl. Acad. Sci. 100: 6388-6393.

Cheng, Z., Yuying, L., Chernhoe, W., Parker, R., and Song, H. 2004. Crystal structure of Ski8p, a WD-repeat protein with dual roles in mRNA metabolism and meiotic recombination. Protein Sci. 13: $2673-2684$.

Christensen, S.K. and Gerdes K. 2003. RelE toxins from bacteria and Archaea cleave mRNAs on translating ribosomes, which are rescued by tmRNA. Mol. Micobiol. 48: 1389-1400.

Christensen, S.K., Pedersen, K., Hansen, F.G., and Gerdes, K. 2003. Toxin-antitoxin loci as stress-response-elements: ChpAK/MazF and ChpBK cleave translated RNAs and are counteracted by tmRNA. J. Mol. Biol. 332: 809-819.

Chiu, S.Y., Serin, G., Ohara, O., and Maquat, L.E. 2003. Characterization of human Smg5/7a: A protein with similarities to Caenorhabditis elegans SMG5 and SMG7 that functions in the dephosphorylation of Upf1. RNA 9: 77-87.

Coburn, G.A. and Mackie, G.A. 1996. Differential sensitivities of portions of the mRNA for ribosomal protein S20 to $3^{\prime}$-exonucleases dependent on oligoadenylation and RNA secondary structure. $J$. Biol. Chem. 271: 15776-15781.

- 1998. Reconstitution of the degradation of the mRNA for ribosomal protein S20 with purified enzymes. J. Mol. Biol. 279: 1061-1074.

Coller, J. and Parker, R. 2004. Eukaryotic mRNA decapping. Аnnu. Rev. Biochem. 73: 861-890.

Cougot, N., Babajko, S., and Seraphin, B. 2004. Cytoplasmic foci are sites of mRNA decay in human cells. J. Cell Biol. 165: 31-40.

Dahlberg, J.E. and Lund, E. 2004. Does protein synthesis occur in the nucleus? Curr. Opin. Cell. Biol. 16: 335-338.

Dahlberg, J.E., Lund, E., and Goodwin, E.B. 2003. Nuclear translation: What is the evidence? RNA 9: 1-8.

Danin-Kreiselman, M., Lee, C.Y., and Chanfreau, G. 2003. RNase III-mediated degradation of unspliced pre-mRNAs and lariat introns. Mol. Cell 11: 1279-1289.

Deikus, G., Babitzke, P., and Bechhofer DH. 2004. Recycling of a regulatory protein by degradation of the RNA to which it binds. Proc. Natl. Acad. Sci. 101: 2747-2751.

Deutscher, M.P. 2003. Degradation of stable RNA in bacteria. J. Biol. Chem. 278: 45041-45044.

Dreyfus, M. and Régnier, P. 2002. The poly(A) tail of mRNAs: Bodyguard in eukaryotes, scavenger in bacteria. Cell 111: 611-613.

Fiedler, T.J., Vincent, H.A., Zuo, Y., Gavrialov, O., and Malhotra, A. 2004. Purification and crystallization of Escherichia coli oligoribonuclease. Acta Crystallogr. D Biol. Crystallogr. 60: 736-739.

Folichon, M., Arluison, V., Pellegrini, O., Huntzinger, E., Régnier, P., and Hajnsdorf, E. 2003. The poly(A) binding protein Hfq protects RNA from RNase E and exoribonucleolytic degradation. Nucleic Acids Res. 31: 7302-7310.
Frischmeyer, P.A., van Hoof, A., O’Donnell, K., Guerrerio, A.L., Parker, R., and Dietz, H.C. 2002. An mRNA surveillance mechanism that eliminates transcripts lacking termination codons. Science 295: 2258-2261.

Gatfield, D. and Izaurralde, E. 2004. Nonsense-mediated messenger RNA decay is initiated by endonucleolytic cleavage in Drosophila. Nature 429: 575-578.

Gatfield, D., Unterholzner, L., Ciccarelli, F.D., Bork, P., Izaurralde, E. 2003. Nonsense-mediated mRNA decay in Drosophila: At the intersection of the yeast and mammalian pathways. EMBO J. 22: 3960-3970.

Gross, J.D., Moerke, N.J., von der Haar, T., Lugovskoy, A.A., Sachs, A.B., McCarthy, J.E., and Wagner, G. 2003. Ribosome loading onto the mRNA cap is driven by conformational coupling between eIF4G and eIF4E. Cell 115: 739-750.

Gu, M., Fabrega, C., Lui, S.W., Liu, H., Kiledjian, M., and Lima, C.D. 2004. Insights into the structure, mechanism, and regulation of scavenger mRNA decapping activity. Mol. Cell 14: 67-80.

Gutierrez, R.A., Ewing, R.M., Cherry, J.M., and Green, P.J. 2002. Identification of unstable transcripts in Arabidopsis by cDNA microarray analysis: Rapid decay is associated with a group of touch- and specific clock-controlled genes. Proc. Natl. Acad. Sci. 99: 1151311518.

Hayes, C.S. and Sauer, R.T. 2003. Cleavage of the A site mRNA codon during ribosome pausing provides a mechanism for translational quality control. Mol. Cell 12: 903-911.

Hayes, C.S., Bose, B., and Sauer, R.T. 2002a. Stop codons preceded by rare arginine codons are efficient determinants of SsrA tagging in Escherichia coli. Proc. Natl. Acad. Sci. 99: 3440-3445.

. 2002b. Proline residues at the $\mathrm{C}$ terminus of nascent chains induce SsrA tagging during translation termination. J. Biol. Chem. 277: 33825-33832.

Hilleren, P. and Parker, R. 1999. Mechanisms of mRNA surveillance in eukaryotes. Annu. Rev. Genet. 33: 229-260.

Ingelfinger, D., Arndt-Jovin, D.J., Luhrmann, R., and Achsel, T. 2002. The human LSm1-7 proteins colocalize with the mRNA-degrading enzymes Dcp1/2 and Xrn1 in distinct cytoplasmic foci. RNA 8: $1489-1501$.

Jackson Jr., J.S., Houshmandi, S.S., Lopez Leban, F., and Olivas, W.M. 2004. Recruitment of the Puf3 protein to its mRNA target for regulation of mRNA decay in yeast. RNA 10: 1625-1636.

Jacobson, A. and Peltz, S.W. 2000. Destabilization of nonsense-containing transcripts in Saccharomyces cerevisiae. In Translational control, 2nd ed. (eds. W.B. Hershey et al.), pp. 827-847. Cold Spring Harbor Laboratory Press, Cold Spring Harbor NY.

Jiang, X. and Belasco, J.G. 2004. Catalytic activation of multimeric RNase E and RNase G by $5^{\prime}$-monophosphorylated RNA. Proc. Natl. Acad. Sci. 101: 9211-9216.

Kadaba, S., Krueger, A., Trice, T., Krecic, A.M., Hinnebusch, A.G., and Anderson, J. 2004. Nuclear surveillance and degradation of hypomodified initiator tRNA ${ }^{\text {met }}$ in S. cerevisiae. Genes \& Dev. 18: 1227 1240.

Kedersha, N. and Anderson, P. 2002. Stress granules: Sites of mRNA triage that regulate mRNA stability and translatability. Biochem. Soc. Trans. 30: 963-969.

Khemici, V. and Carpousis, A.J. 2004. The RNA degradosome and poly(A) polymerase of Escherichia coli are required in vivo for the degradation of small mRNA decay intermediates containing REPstabilizers. Mol. Microbiol. 51: 777-790.

Kushner, S. 2002. mRNA decay in Escherichia coli comes of age. J. Bacteriol. 184: 4658-4665.

Kwak, J.E., Wang, L., Ballantyne, S., Kimble, J., and Wickens, M. 2004. Mammalian GLD-2 homologs are poly(A) polymerases. Proc. Natl. Acad. Sci. 101: 4407-4412.

Lai, W.S., Kennington, E.A., and Blackshear, P.J. 2003. Tristetraprolin and its family members can promote the cell-free deadenylation of AU-rich elements-containing mRNAs by poly(A) ribonuclease. Mol. Cell. Biol. 23: 3798-3812.

Lamontagne, B., Hannoush, R.N., Damha, M.J., and Abou Elela, S. 
2004. Molecular requirements for duplex recognition and cleavage by eukaryotic RNase III: Discovery of an RNA-dependent DNA cleavage activity of yeast Rntlp. J. Mol. Biol. 338: 401-418.

Le Derout, J., Folichon, M., Briani, F., Deho, G., Régnier, P., and Hajnsdorf, E. 2003. Hfq affects the length and the frequency of short oligo(A) tails at the $3^{\prime}$ end of Escherichia coli rpsO mRNAs. Nucleic Acids Res. 31: 4017-4023.

Lee, K., Zhan, X., Gao, J., Qiu, J., Feng, Y., Meganathan, R., Cohen, S.N., and Georgiou, G. 2003. RraA, a protein inhibitor of RNase E activity that globally modulates RNA abundance in E. coli. Cell 114: 623-634.

Li, Z., Pandit, S.P., and Deutscher, M.P. 1998. Polyadenylation of stable RNA precursors in vivo. Proc. Natl. Acad. Sci. 95: 1215812162.

Li, Z., Reimers, S., Pandit, S., and Deutscher, M.P. 2002. RNA quality control: Degradation of defective transfer RNA. EMBO J. 21: 11321138.

Liu, H., Rodgers, N.D., Jiao, X., and Kiledjian, M. 2002. The scavenger mRNA decapping enzyme DcpS is a member of the HIT family of pyrophosphatases. EMBO J. 21: 4699-4708.

Liu, S., Jiao, X., Liu, H., Gu, M., Lima, C., and Kiledjian, M. 2004. Functional analysis of mRNA scavenger decapping enzymes. RNA 10: $1412-1422$.

Mackie, G.A. 1998. Ribonuclease E is a 5'-end-dependent endonuclease. Nature 395: 720-723.

. 2000. Stabilization of circular rpsT mRNA demonstrates the $5^{\prime}$-end dependence of RNase $\mathrm{E}$ action in vivo. J. Biol. Chem. 275: 25069-25072.

Maquat, L.E. 2002. NASty effects on fibrillin pre-mRNA splicing: Another case of ESE does it, but proposals for translation-dependent splice site choice live on. Genes \& Dev. 16: 1743-1753.

. 2004. Nonsense-mediated mRNA decay: Splicing, translation and mRNP dynamics. Nat. Rev. Mol. Cell. Biol. 5: 89-99.

Marzluff, W.F. and Duronio, R.J. 2002. Histone mRNA expression: Multiple levels of cell cycle regulation and important developmental consequences. Curr. Opin. Cell. Biol. 14: 629-699.

Medghalchi, S.M., Frischmeyer, P.A., Mendell, J.T., Kelly, A.G., Lawler, A.M., and Dietz, H.C. 2001. Rent1, a trans-effector of nonsense-mediated mRNA decay, is essential for mammalian embryonic viability. Hum. Mol. Genet. 10: 99-105.

Mohanty, B.K. and Kushner, S.R. 2004. The Sm-like protein Hfq regulates polyadenylation-dependent mRNA decay in Escherichia coli. Mol. Microbiol. (in press).

Moll, I., Afonyushkin, T., Vytvytska, O., Kaberdin, V.R., and Blasi, U. 2003. Coincident Hfq binding and RNase E cleavage sites on mRNA and small regulatory RNAs. RNA 9: 1308-1314.

Monzingo, A.F., Gao, J., Qiu, J., Georgiou, G., and Robertus, J.D. 2003. The X-ray structure of Escherichia coli RraA (MenG), a protein inhibitor of RNA processing. J. Mol. Biol. 332: 1015-1024.

Mühlemann, O., Mock-Casagrande, C.S., Wang, J., Li, S., Custodio, N., Carmo-Fonseca, M., Wilkinson, M.F., and Moore, M.J. 2001. Precursor RNAs harboring nonsense codons accumulate near the site of transcription. Mol. Cell 8: 33-43.

Page, M.F., Carr, B., Anders, K.R., Grimson, A., and Anderson, P. 1999. SMG-2 is a phosphorylated protein required for mRNA surveillance in Caenorhabditis elegans and related to Upflp of yeast. Mol. Cell. Biol. 19: 5943-5951.

Parker, R. and Song, H. 2004. The enzymes and control of eukaryotic mRNA turnover. Nat. Struct. Mol. Biol. 11: 121-127.

Pastori, R.L., Moskaitis, J.E., Buzek, S.W., and Schoenberg, D.R. 1991a. Coordinate estrogen-regulated instability of serum proteincoding messenger RNAs in Xenopus laevis. Mol. Endorcrinol. 5: 461-468.

Pastori, R.L., Moskaitis, J.E., and Schoenberg, D.R. 1991b. Estrogeninduced ribonuclease activity in Xenopus liver. Biochemistry 30: 10490-10498.

Pedersen, K., Zavialov, A.V., Pavlov, M.Y., Elf, J., Gerdes, K., and Ehrenberg, M. 2003. The bacterial toxin RelE displays codon-specific cleavage of mRNA in the ribosomal A site. Cell 112: 131-140.
Perez-Amador, M.A., Lidder, P., Johnson, M.A., Landgraf, J., Wisman, E., and Green, P.J. 2001. New molecular phenotypes in the dst mutants of Arabidopsis revealed by DNA microarray analysis. Plant Cell 13: 2703-2713.

Pham, J.W., Pellino, J.L., Lee, Y.S., Carthew, R.W., and Sontheimer, E.J. 2004. A Dicer-2-dependent $80 \mathrm{~S}$ complex cleaves targeted mRNAs during RNAi in Drosophila. Cell 117: 83-94.

Raghavan, A., Robison, R.L., McNabb, J., Miller, C.R., Williams, D.A., and Bohjanen, P.R. 2001. HuA and tristetraprolin are induced following $\mathrm{T}$ cell activation and display distinct but overlapping RNA binding specificities. J. Biol. Chem. 276: 47958-47965.

Ryan, C.M., Militello, K.T., and Read, L.K. 2003. Polyadenylation regulates the stability of Trypanosoma brucei mitochondrial RNAs. J. Biol. Chem. 278: 32753-32762.

Schoenberg, D.R. and Chernokalskaya, E. 1997. Ribonucleases involved in eukaryotic mRNA turnover. In mRNA metabolism and post-transcriptional gene regulation (eds. J. Harford and D.R. Morris), pp. 217-240. Wiley, New York.

She, M., Decker, C.J., Sundramurthy, K., Liu, Y., Chen, N., Parker, R., and Song, H. 2004. Crystal structure of Dcplp and its functional implications in mRNA decapping. Nat. Struct. Mol. Biol. 11: 249256.

Sheth, U. and Parker, R. 2003. Decapping and decay of messenger RNA occur in cytoplasmic processing bodies. Science 300: 805-808.

Singh, G. and Lykke-Andersen, J. 2003. New insights into the formation of active nonsense-mediated decay complexes. Trends Biochem. Sci. 28: 464-466.

Souret, F.F., Kastenmayer, J.P., and Green, P.J. 2004. AtXRN4 degrades mRNA in Arabidopsis and its substrates include selected miRNA targets. Mol. Cell 15: 173-183.

Stoecklin, G., Stubbs, T., Kedersha, N., Wax, S., Rigby, W.F., Blackwell, T.K., and Anderson, P. 2004. MK2-induced tristetraprolin: 14-3-3 complexes prevent stress granule association and AREmRNA decay. EMBO J. 23: 1313-1324.

Stumpo, D.J., Byrd, N.A., Phillips, R.S., Ghosh, S., Maronpot, R.R., Castranio, T., Meyers, E.N., Mishina, Y., and Blackshear, P.J. 2004. Chorioallantoic fusion defects and embryonic lethality resulting from disruption of $\mathrm{Zfp} 36 \mathrm{~L} 1$, a gene encoding a CCCH tandem zinc finger protein of the Tristetraprolin family. Mol. Cell. Biol. 24: 6445-6455.

Sunohara, T., Abo, T., Inada, T., and Aiba, H. 2002. The C-terminal amino acid sequence of nascent peptide is a major determinant of SsrA tagging at all three stop codons. RNA 8: 1416-1427.

Sunohara, T., Jojima, K., Tagami, H., Inada, T., and Aiba, H. 2004a. Ribosome stalling during translation elongation induces cleavage of mRNA being translation in Escherichia coli. J. Biol. Chem. 279: 15368-15375.

Sunohara, T., Jojima, K., Yamamoto, Y., Inada, T., and Aiba, H. 2004b. Nascent-peptide-mediated ribosome stalling at a stop codon induces mRNA cleavage resulting in nonstop mRNA that is recognized by tmRNA. RNA 10: 378-386.

Temme, C., Zaessinger, S., Meyer, S., Simonelig, M., and Wahle, E. 2004. A complex containing the CCR4 and CAF1 proteins is involved in mRNA deadenylation in Drosophila. EMBO J. 23: 28622871.

Tucker, M., Valencia-Sanchez, M.A., Staples, R.R., Chen, J., Denis, C.L., and Parker, R. 2001. The transcription factor associated Ccr4 and Cafl proteins are components of the major cytoplasmic deadenylase in Saccharomyces cerevisiae. Cell 104: 377-386.

Tucker, M., Staples, R.R., Valencia-Sanchez, M.A., Muhlrad, D., Parker, R. 2002. Ccr4p is the catalytic subunit of a Ccr4p/Pop2p/ Notp mRNA deadenylase complex in Saccharomyces cerevisiae. EMBO J. 21: 1427-1436.

Tzivion, G. and Avruch, J. 2002. 14-3-3 proteins: Active cofactors in cellular regulation by serine/threonine phosphorylation. J. Biol. Chem. 277: 3061-3064.

van Dijk, E., Cougot, N., Meyer, S., Babajko, S., Wahle, E., Saraphin, B. 2002. Human Dcp2: A catalytically active mRNA decapping enzyme located in specific cytoplasmic structures. EMBO J. 


\section{1: 6915-6924.}

van Hoof, A., Frischmeyer, P.A., Dietz, H.C., and Parker, R. 2002. Exosome-mediated recognition and degradation of mRNAs lacking a termination codon. Science 295: 2262-2264.

Vecerek, B., Moll, I., Afonyushkin, T., Kaberdin, V., and Blasi, U. 2003. Interaction of the RNA chaperone Hfq with mRNAs: Direct and indirect roles of $\mathrm{Hfq}$ in iron metabolism of Escherichia coli. Mol. Microbiol. 50: 897-909.

Volpe, T.A., Kidner, C., Hall, I.M., Teng, G., Grewal, S.I., and Martienssen, R.A. 2002. Regulation of heterochromatic silencing and histone H3 lysine-9 methylation by RNAi. Science 297: 1833-1837.

von der Haar, T., Gross, J.D., Wagner, G., McCarthy, J.E. 2004. The mRNA cap-binding protein eIF4E in post-transcription gene expression. Nat. Struct. Mol. Biol. 11: 503-511.

Vytvytska, O., Jakobsen, J.S., Balcunaite, G., Andersen, J.S., Baccarini, M., and von Gabain, A. 1998. Host factor I, Hfa, binds to Escherichia coli ompA mRNA in a growth rate-dependent fashion and regulates its stability. Proc. Natl. Acad. Sci. 95: 18-23.

Vytvytska, O., Moll, I., Kaberdin, V.R., von Gabain, A., and Blasi, U. 2000. Hfq (HF1) stimulates ompA mRNA decay by interfering with ribosome binding. Genes \& Dev. 14: 1109-1118.

Wang, J., Chang, Y.F., Hamilton, J.U.I., and Wilkinson, M.F. 2002a. Nonsense-associated altered splicing: A frame-dependent response distinct from nonsense-mediated decay. Mol. Cell 10: 951-957.
Wang, L., Eckmann, C.R., Kadyk, L.C., Wickens, M., and Kimble, J. 2002b. A regulatory cytoplasmic poly(A) polymerase in Caenorhabditis elegans. Nature 419: 312-316.

Wickens, M., Bernstein, D.S., Kimble, J., and Parker, R. 2002. A PUF family portrait: $3^{\prime}$ UTR regulation as a way of life. Trends Genet. 18: $150-157$.

Wilkinson, M.F. and Shyu, A.B. 2002. RNA surveillance by nuclear scanning? Nat. Cell. Biol. 4: 144-147.

Withey, J.H. and Friedman, D.I. 2003. A salvage pathway for protein structures: tmRNA and trans-translation. Annu. Rev. Microbiol. 57: 101-123.

Wu, H., Yang, P.K., Butcher, S.E., Kang, S., Chanfreau, G., and Feigon, J. 2001. A novel family of RNA tetraloop structure forms the recognition site for Saccharomyces cerevisiae RNase III. EMBO J. 20: 7240-7249.

Yaffe, M.B. 2002. How do 14-3-3 proteins work?-Gatekeeper phosphorylation and the molecular anvil hypothesis. FEBS Lett. 513: 53-57.

Yang, F. and Schoenberg, D.R. 2004. Endonuclease-mediated mRNA decay involves the selective targeting of PMR1 to polyribosomebound substrate mRNA. Mol. Cell 14: 435-445.

Zilberman, D., Cao, X., and Jacobsen, S.E. 2003. ARGONAUTE4 control of locus-specific siRNA accumulation and DNA and histone methylation. Science 299: 716-719. 

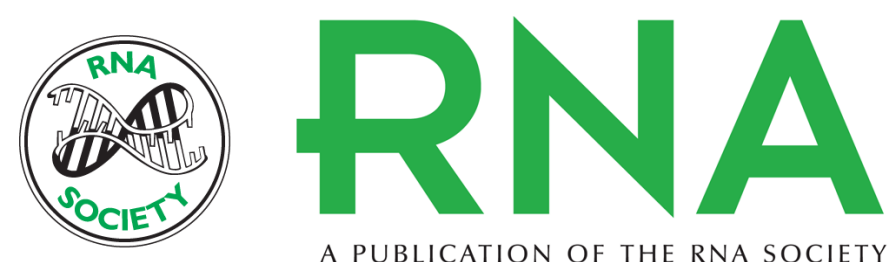

A PUBLICATION OF THE RNA SOCIETY

\title{
Under the Tucson sun: A meeting in the desert on mRNA decay
}

\author{
KRISTIAN E. BAKER and CIARÁN CONDON
}

RNA 2004 10: 1680-1691

References This article cites 97 articles, 46 of which can be accessed free at:

http://rnajournal.cshlp.org/content/10/11/1680.full.html\#ref-list-1

License

Email Alerting Receive free email alerts when new articles cite this article - sign up in the box at the Service top right corner of the article or click here. 